\title{
Protein Phosphatase 1-Dependent Bidirectional Synaptic Plasticity Controls Ischemic Recovery in the Adult Brain
}

\author{
Gaël F. Hédou, ${ }^{1 \star}$ Kyoko Koshibu, ${ }^{1 \star}$ Mélissa Farinelli, ${ }^{1}$ Ertugrul Kilic, ${ }^{2}$ Christine E. Gee, ${ }^{1}$ Ulkan Kilic, ${ }^{2}$ \\ Karsten Baumgärtel, ${ }^{1}$ Dirk M. Hermann, ${ }^{2}$ and Isabelle M. Mansuy ${ }^{1}$ \\ ${ }^{1}$ Brain Research Institute, Medical Faculty, University of Zurich and Department of Biology, Swiss Federal Institute of Technology, CH-8057 Zurich, \\ Switzerland, and ${ }^{2}$ Department of Neurology, University Hospital Zurich, CH-8091 Zurich, Switzerland
}

\begin{abstract}
Protein kinases and phosphatases can alter the impact of excitotoxicity resulting from ischemia by concurrently modulating apoptotic/ survival pathways. Here, we show that protein phosphatase 1 (PP1), known to constrain neuronal signaling and synaptic strength (Mansuy et al., 1998; Morishita et al., 2001), critically regulates neuroprotective pathways in the adult brain. When PP1 is inhibited pharmacologically or genetically, recovery from oxygen/glucose deprivation (OGD) in vitro, or ischemia in vivo is impaired. Furthermore, in vitro, inducing LTP shortly before OGD similarly impairs recovery, an effect that correlates with strong PP1 inhibition. Conversely, inducing LTD before OGD elicits full recovery by preserving PP1 activity, an effect that is abolished by PP1 inhibition. The mechanisms of action of PP1 appear to be coupled with several components of apoptotic pathways, in particular ERK1/2 (extracellular signal-regulated kinase 1/2) whose activation is increased by PP1 inhibition both in vitro and in vivo. Together, these results reveal that the mechanisms of recovery in the adult brain critically involve PP1, and highlight a novel physiological function for long-term potentiation and long-term depression in the control of brain damage and repair.
\end{abstract}

Key words: PP1; ischemia; OGD; cell death; plasticity; hippocampus

\section{Introduction}

Cerebral ischemia often leads to neural damage resulting from transient deprivation of oxygen and nutrients in the brain. Such deprivation causes a dramatic increase in neuronal excitation caused by enhanced glutamate release and a toxic rise in intracellular calcium $\left(\mathrm{Ca}^{2+}\right)$ (Olney and Sharpe, 1969; Arundine and Tymianski, 2004). Affected regions of the brain are often irreversibly injured and show long-lasting functional impairments. Because ischemia is highly detrimental to brain functions, much work has been done to better understand the underlying molecular mechanisms and to develop neuroprotective therapies. The limited success of glutamate receptor (NMDA or AMPA) antagonists in the clinic (Aarts and Tymianski, 2003) has, however,

\footnotetext{
Received Sept. 11, 2007; revised Nov. 10, 2007; accepted Nov. 10, 2007.

This work was supported by the University of Zurich (laboratory of I.M.M.), the Swiss Federal Institute of Technology (laboratory of I.M.M.), the National Center for Competence in Research "Neural Plasticity and Repair" (laboratories of I.M.M. and D.M.H.), the Swiss National Science Foundation (laboratories of I.M.M. and D.M.H.), the Human Frontier Science Program (laboratory of I.M.M.), European Molecular Biology Organization (laboratory of I.M.M.), the Novartis Research Foundation (laboratory of I.M.M.), The Slack Gyr Foundation (laboratory of I.M.M.), the Center of Integrative Human Physiology (laboratory of D.M.H.), and the Swiss Heart Foundation (laboratory of D.M.H.). We thank Beat Gähwiler, Urs Gerber, Kaspar Vogt, and Dominique Muller for help and advice, Hansjörg Kasper and Angela Fendel for technical support, and Gregor Fisher for help with the mouse colony.

* G.F.H. and K.K. contributed equally to this work.

Correspondence should be addressed to Isabelle M. Mansuy, Brain Research Institute, Medical Faculty, University of Zurich and Department of Biology, Swiss Federal Institute of Technology, Winterthurerstrasse 190, CH-8057 Zurich, Switzerland. E-mail: mansuy@hifo.unizh.ch.

G. F. Hédou's present address: GlaxoSmithKline, Psychiatry Centre of Excellence for Drug Discovery, Laboratory of Behavioral Neurochemistry, Via Fleming 4, 37135 Verona, Italy.

C. E. Gee's present address: Novartis Institutes for Biomedical Research, Novartis Pharma AG, CH-4002 Basel, Switzerland.

DOI:10.1523/JNEUROSCI.4109-07.2008

Copyright $\odot 2008$ Society for Neuroscience $\quad 0270-6474 / 08 / 280154-09 \$ 15.00 / 0$
}

prompted interest in the intracellular pathways downstream from glutamate receptors, with the hope that their study would bring potential therapeutic insight (Legos et al., 2002; Aarts and Tymianski, 2003).

$\mathrm{Ca}^{2+}$ overload during ischemic insult dramatically alters $\mathrm{Ca}^{2+}$-dependent signaling and buffering systems (Lipton, 1999; Sattler and Tymianski, 2000; Arundine and Tymianski, 2003; Sugawara et al., 2004) and perturbs the activity of $\mathrm{Ca}^{2+}$ dependent protein kinases and phosphatases. Such alteration strongly impacts the outcome of excitotoxicity. Thus, an imbalance between the activity of the $\mathrm{Ca}^{2+}$-dependent protein kinase II (CaMKII), protein kinase C (PKC) (Onodera et al., 1995; Aronowski et al., 2000), and the protein phosphatase calcineurin (CaN or PP2B) in favor of $\mathrm{CaN}$, induces an excessive dephosphorylation of proapoptotic proteins (Asai et al., 1999; Wang et al., 1999) and leads to delayed neuronal death. In contrast, inhibition of CaN by the immunosuppressants FK506 (Fujimycin) or cyclosporine A prevents neuronal degeneration, reduces infarct size, and rescues motor functions after experimental stroke in rat (Sharkey and Butcher, 1994; Sharkey et al., 1996; Morioka et al., 1999). These results therefore indicate that $\mathrm{CaN}$ has both apoptotic and neuroprotective functions that may depend on the nature, kinetics, and extent of the injury.

Unlike CaN, the functions of other brain protein phosphatases in cerebral ischemia remain mostly unknown. In particular, the role of protein phosphatase 1 (PP1), the second most abundant protein serine/threonine phosphatase in the brain, has not been extensively examined in the context of brain injury. A few reports in culture systems, however, have suggested that PP1 may have neuroprotective functions (Fernandez et al., 1993; Nuydens 
et al., 1998; Runden et al., 1998; Klumpp and Krieglstein, 2002; Yi et al., 2005). To examine the potential functions of PP1 in the mechanisms of recovery, we used in vitro and in vivo models of ischemia in the adult mouse brain, and tested the effect of a change in PP1 activity on recovery. Pharmacological, genetic, and electrophysiological methods were used to inhibit or activate PP1 in vitro and in vivo, and recovery was evaluated after oxygen/ glucose deprivation (OGD) in hippocampal slices or focal middle cerebral artery occlusion (MCAO) in adult mice. Our results demonstrate that PP1 and PP1-dependent bidirectional plasticity control the mechanisms of recovery in the adult brain, and that PP1 involves downstream components of apoptotic and cell survival pathways, in particular extracellular signal-regulated kinase $1 / 2$ (ERK1/2), both in vitro and in vivo.

\section{Materials and Methods}

Animals. Adult C57BL/6J mice or I- $1^{\star}$ mutant and control littermates $\left(9-12\right.$ weeks of age) were used. I- $1^{\star}$ mice were obtained as previously described (Genoux et al., 2002) and were backcrossed to the C57BL/6J background for at least $8-10$ generations. Mutant mice carry the CaMKII $\alpha$ promoter-rtTA(2) and tetO-I- $1^{*}$ transgenes, and control mice are littermates carrying no transgene or either one of the transgenes. Mice were fed with $6 \mathrm{mg} / \mathrm{g}$ doxycycline (Westward Pharmaceuticals, Eatontown, NJ) mixed with wet food (50:50 food-to-water ratio) for at least $7 \mathrm{~d}$ before experiments. All experiments were performed in accordance with Swiss Federal Veterinary Office regulations and by experimenters blind to genotype.

Electrophysiological recordings. Adult mice were killed by cervical dislocation, and heads were immediately immersed for at least $3 \mathrm{~min}$ in freshly prepared ice-cold oxygenated artificial CSF (aCSF) (119 mM $\mathrm{NaCl}, 11 \mathrm{~mm}$ D-glucose, $1.3 \mathrm{~mm} \mathrm{MgCl}_{2} \cdot 6 \mathrm{H}_{2} \mathrm{O}, 1.3 \mathrm{~mm} \mathrm{NaH}_{2} \mathrm{PO}_{4}, 2.5 \mathrm{~mm}$ $\mathrm{KCl}, 2.5 \mathrm{~mm} \mathrm{CaCl}_{2}, 26 \mathrm{mM} \mathrm{NaHCO}_{3}$, gassed for a minimum of $20 \mathrm{~min}$ before use and throughout experiment with $95 \% \mathrm{O}_{2} / 5 \% \mathrm{CO}_{2}$ ). Brains were quickly removed, and hippocampi were dissected. Acute slices (400 $\mu \mathrm{m}$ thick) were prepared with a vibratome (VT 1000S; Leica, Nussloch, Germany) in ice-cold oxygenated aCSF. Slices were transferred to an interface chamber at $34^{\circ} \mathrm{C}$ for $40-50 \mathrm{~min}$, and then kept at room temperature for at least $1-2 \mathrm{~h}$ before recording. Recordings were performed at $27-28^{\circ} \mathrm{C}$ in a submersion chamber (Slice Mini Chamber III/IV; Luigs \& Neumann, Ratingen, Germany) continuously perfused with oxygenated aCSF at $1.1 \mathrm{ml} / \mathrm{min}$. A monopolar electrode was placed in the stratum radiatum to activate Schaffer collaterals and test stimuli were applied at $0.033 \mathrm{~Hz}$ at an intensity set to evoke one-third to one-half of the maximum field EPSP (f-EPSP). Evoked f-EPSPs were recorded in the stratum radiatum with a borosilicate micropipette filled with $3 \mathrm{M} \mathrm{NaCl}$ or aCSF. Signals were amplified with an AXOPATCH 200B amplifier (Molecular Devices, Palo Alto, CA) and sampled using pCLAMP. Baseline responses were recorded until stable for at least $10 \mathrm{~min}$.

OGD. Acute slices (hippocampus, cortex, and striatum) were exposed to 15 to $25 \mathrm{~min}$ of hypoxic/aglycemic conditions by perfusion with aCSF containing no glucose (replaced with equimolar sucrose) and gassed with $\mathrm{N}_{2} / \mathrm{CO}_{2}(95 \% / 5 \%)$. Shorter OGD ( $\left.\geq 15 \mathrm{~min}\right)$ was applied in slices when $0 \mathrm{mV}$ f-EPSP was reached before $25 \mathrm{~min}$ incubation. The results from these experiments were not statistically different from those obtained with 25 min OGD and were therefore pooled for final analyses. OGD solution reached the recording chamber within $30 \mathrm{~s}$ and replaced entirely the normoxic aCSF within 2-3 min.

Long-term potentiation/long-term depression. Long-term potentiation (LTP) was induced by a $1 \mathrm{~s}$ train at $100 \mathrm{~Hz}$, and long-term depression (LTD) by $10 \mathrm{~min}$ paired pulse stimulation at $2 \mathrm{~Hz}$ with $200 \mathrm{~ms}$ interstimuli interval, applied to the Schaffer collaterals. In experiments combining LTP or LTD with OGD, normoxic aCSF was replaced with OGD aCSF $10 \mathrm{~min}$ after the end of LTP or LTD induction. Two-pathway experiments were performed by placing stimulating electrodes in stratum radiatum on either side of the recording electrode. Alternating test stimuli were given every 15 or $7.5 \mathrm{~s}$ for LTP or LTD experiments, respectively. High-frequency stimuli (HFS) or low-frequency stimuli (LFS) were applied on alternating electrodes to ensure that both pathways could be modulated. Conditioning stimuli were delivered to both pathways in slices used for the phosphatase assay.

Treatment and drugs. Drugs for slice recordings were bath-applied for $2 \mathrm{~h}$ before recording in oxygenated aCSF. Okadaic acid (OA) (Alexis Corporation, Lausen, Switzerland) and tautomycin (Alexis Corporation; Sigma, Buchs, Switzerland) were dissolved in DMSO (30 mM) and methanol or ethanol (1 mM), respectively, and stock solutions were diluted further in aCSF the day of the experiment. The respective vehicles were used for drug-control experiments. For both mutant and control slices, aCSF was supplemented with doxycycline hydrochloride (Sigma) at 8 $\mathrm{ng} / \mathrm{ml}$ and kept protected from direct light illumination.

Phosphatase assay. Hippocampal slices were stimulated on two pathways with HFS or LFS 10 min before OGD as described above. CA1 was dissected either immediately (OGD $0 \mathrm{~min}$ ) or $1 \mathrm{~h}$ (OGD $1 \mathrm{~h}$ ) after OGD, homogenized in buffer [3.75 mm Tris-HCl, pH 7.4, 15 mm KCl, 3.75 mm $\mathrm{NaCl}, 250 \mu \mathrm{M}$ EDTA, $50 \mu \mathrm{M}$ EGTA, 30\% (v/v) glycerol, $15 \mathrm{~mm}$ $\beta$-mercaptoethanol, protease inhibitor mixture (Sigma), $100 \mu \mathrm{M}$ PMSF] using a 26 gauge syringe, and frozen on dry ice or in liquid nitrogen. Phosphatase activity was determined by incubating $\sim 2 \mu \mathrm{g}$ sample with $0.15 \mathrm{~mm}$ RII phosphopeptide substrate and $5 \mathrm{~nm}$ tautomycin (for PP1 activity) or $5 \mathrm{~nm}$ tautomycin/5 nM OA (for PP1 and PP2A activity) in buffer containing $50 \mathrm{~mm}$ Tris- $\mathrm{HCl}$, pH 7.0, $100 \mu \mathrm{M} \mathrm{Na} \mathrm{N}_{2}$ EDTA, $5 \mathrm{~mm}$ DTT, and $0.01 \%$ Brij 35 at $30^{\circ} \mathrm{C}$ for $10 \mathrm{~min}$. RII is a phosphopeptide derived from the regulatory subunit of PKA (protein kinase A) that is phosphorylated on serine residues and thus can be dephosphorylated by any of the serine/threonine phosphatases. The specificity of the measurement of PP1 and PP2A activity relies on the specificity of the pharmacological phosphatase inhibitors, tautomycin and okadaic acid (do not inhibit PP2C) at defined doses. The reaction was terminated by addition of TCA and centrifugation at $13,000 \times g$ for $5 \mathrm{~min}$. The amount of free phosphates released in the reaction was measured by mixing the supernatant with Biomol Green reagent (Biomol, Plymouth Meeting, PA) and color density was detected at $\mathrm{OD}_{620}$. For determining total phosphatase activity, tautomycin and OA were removed from the reaction. Background activity was measured separately in a reaction without RII substrate and in a reaction without sample. The percentage phosphatase activity was calculated by subtracting the background from the total and inhibited reactions, and then dividing the inhibited activity by the total activity.

Intraluminal MCAO. Adult I-1* mutant and control littermates (21-28 g) were anesthetized with isoflurane and subjected to focal cerebral ischemia by intraluminal MCAO using an 8-0 silicon-coated (Xantopren; Bayer Dental, Osaka, Japan) nylon monofilament (Ethilon; Ethicon, Norderstedt, Germany). Laser Doppler flow was monitored using a flexible $0.5 \mathrm{~mm}$ fiberoptic probe (Perimed, Stockholm, Sweden) attached to the intact skull overlying the middle cerebral artery territory. Rectal temperature was maintained at $36.5-37.0^{\circ} \mathrm{C}$ with a feedback-controlled heating system. After MCAO, wounds were carefully sutured and anesthesia was discontinued. After $24 \mathrm{~h}$ (90 min MCAO) or $72 \mathrm{~h}(30 \mathrm{~min}$ $\mathrm{MCAO}$ ) of reperfusion, animals were anesthetized with isoflurane and killed. Brain was removed and either frozen on dry ice and cut into $18 \mu \mathrm{m}$ cryostat sections or dissected out bilaterally for Western blot analyses.

Histology. Brain sections from animals subjected to $90 \mathrm{~min}$ of MCAO were fixed in $4 \%$ paraformaldehyde/ $0.1 \mathrm{M}$ PBS and infarct volumetry was performed after cresyl violet staining. Five sections from equidistant brain levels ( $2 \mathrm{~mm}$ apart) were evaluated for each animal. Borders between infarcted and noninfarcted tissue were outlined using an image analysis system (Image J) (Spudich et al., 2006) by an investigator blind to genotype outlining the unaffected (i.e., healthy tissue on both sides of the brain), from which the difference value was calculated, which reflects the edema-corrected infarct area on a particular brain level. The infarct areas from all levels of the brain $(n=4)$ were finally integrated over the whole brain for determining infarct volume. In animals undergoing $30 \mathrm{~min}$ $\mathrm{MCAO}$, section from the bregma level were stained by terminal deoxynucleotidyl transferase-mediated biotinylated UTP nick end labeling (TUNEL) using a kit (Roche, Basel, Switzerland). The number of DNA fragmented cells in the striatum was quantified by a stereological method using uniform random sampling of an array with six regions of interest 
( $250 \times 250 \mu \mathrm{m}$ per area) separated by $250 \mu \mathrm{m}$, for which mean values were calculated to determine the number of irreversibly injured cells as previously described (Hermann et al., 2001).

Western blots. Tissue samples from the striatum ipsilateral and contralateral to MCAO or from acute forebrain slices (hippocampus, cortex, and striatum) were homogenized and centrifuged. Supernatants were resolved on SDS-PAGE, and proteins were transferred onto polyvinylidene difluoride membranes. Membranes were dried and incubated in blocking solution, and then in rabbit anti-ERK1/2 (9102; Cell Signaling, Beverly, MA), mouse anti-phospho-ERK1/2 (anti-phospho-Thr and Tyr, M8159; Sigma), rabbit anti-c-Jun N-terminal kinase 1/2 (JNK1/2) (JNK2; sc-572; Santa Cruz Biotechnology, Santa Cruz, CA), rabbit antiphospho-JNK1/2 (anti-Tyr185, 9251; Cell Signaling), rabbit anti-Bcl-X (610212; BD Biosciences, Mountain View, CA), or rabbit anti-activated caspase-3 (CM1; BD Biosciences) antibody (1:500 in 0.1\% Tween 20, 0.1 м TBS) (Kilic et al., 2005). Membranes were rinsed, incubated in peroxidase-coupled secondary antibody $(1: 2000$ in $0.1 \%$ Tween $20,0.1 \mathrm{M}$ TBS), washed, immersed in enhanced chemoluminescence (ECL) solution, and then exposed to ECL-Hyperfilm (Amersham Biosciences, Piscataway, NJ). The band intensity was determined by densitometry and corrected for protein loading using anti- $\beta$-actin antibody (A5316; Sigma) as an internal control.

Data analysis. For each electrophysiological experiment, the average slope of individual f-EPSP was measured from the initial 1-1.5 ms portion after the fiber volley using Clampfit (Molecular Devices). For statistical analyses, the f-EPSP slope was averaged over $1 \mathrm{~min}$ and analyzed by three-way ANOVA using Statview 5.0 with sequences ( $10 \mathrm{~min}$ ) and stimulation (1 min bins) as repeated measures. The between-subjects factors were OA doses (control vs $0.1 \mathrm{nM}, 1 \mathrm{nM}$, or $1 \mu \mathrm{M}$ ), tautomycin doses (control vs $1,5,10$, or $100 \mathrm{~nm}$ ), genotype (controls vs mutants), delay between LTP or LTD and OGD (controls vs 10 or $30 \mathrm{~min}$ ), genotype on LTD and LTD plus OGD (controls vs mutants) and combined genotypedrug treatment on LTD and LTD plus OGD (controls vs mutant plus tautomycin). Unpaired $t$ tests were performed on mean f-EPSP for 10 min toward the end of recording as indicated in Results, when main effect of between-subjects factor reached significance. In some cases, the generalized linear model followed by Tukey's or least significant difference (LSD) post hoc tests were used to evaluate electrophysiological or phosphatase assays data. Infarct volume and DNA fragmentation were analyzed by two-tailed $t$ tests. Western blots were evaluated by one-way ANOVA followed by LSD post hoc tests. Statistical significance was set at $p=0.05$, and data are presented as mean \pm SEM.

\section{Results}

\section{Pharmacological and genetic inhibition of PP1 reduces recovery from OGD in acute hippocampal slices}

To examine the role of PP1 in the mechanisms of recovery from ischemic injury, we simulated conditions of transient cerebral ischemia in vitro by exposing acute hippocampal slices from adult mouse brain to OGD. Unlike long-term deprivation that causes irreversible cell death, transient OGD induces mild and reversible damage such as decreased metabolic activity (Lushnikova et al., 2004), inhibition of transmitter release (Fowler, 1989), and increased postsynaptic $\mathrm{Ca}^{2+}$ influx, spontaneous miniature EPSC amplitude, evoked AMPA and NMDA receptor-mediated excitatory responses, and AMPA/NMDA responses ratio (Quintana et al., 2006). The impact of OGD was evaluated by measuring f-EPSPs evoked in area CA1 by electrical stimulation of Schaffer collaterals (Raley-Susman and Lipton, 1990; Lobner and Lipton, 1993). OGD induced a rapid decline of the f-EPSP slope, followed by a partial recovery, and then a slow stabilization to, however, a level that was significantly lower than baseline. These results indicate that OGD induces long-lasting cellular changes that decrease $\mathrm{f}$-EPSP and are indicative of damaged neuronal functions (Fig. 1A, solid circles). We then examined the impact of PP1 inhibition before OGD using pharmacological, genetic, and physiological means on recovery. First, tautomycin, a highly se- lective PP1 inhibitor $\left(\mathrm{IC}_{50}\right.$ for PP1, 1.1-7.5 nM; for PP2A, 10-23 nM) was applied to slices before OGD, at a dose ranging from 1 to $100 \mathrm{~nm}$ to inhibit either PP1 alone (low concentration) or both PP1 and PP2A (high concentration). A significant dosedependent reduction in f-EPSP recovery was observed (dose effect, $F_{(5,20)}=139.8, p<0.001$ ) (Fig. $1 A$ ), which had an $\mathrm{IC}_{50}$ of 2.4 nм (95\% confidence, 2.2-2.7 nM) (Fig. $1 B$ ), strongly suggesting that it was mediated by PP1 inhibition $\left(\mathrm{IC}_{50}<10 \mathrm{nM}\right.$ ) and not by PP2A inhibition [ $\mathrm{IC}_{50}>10 \mathrm{~nm}$ (Gupta et al., 1997)]. To confirm these results, we used $\mathrm{OA}(0.1 \mathrm{nM}$ to $1 \mu \mathrm{M})$, another protein phosphatase inhibitor 50-fold more selective for PP2A than PP1 [ $\mathrm{IC}_{50}$ for PP2A, 0.02-1.0 nм; for PP1, 10-1300 nM (Gupta et al., 1997)]. We reasoned that, if the effect of tautomycin is attributable to PP1 inhibition, low doses of OA that only inhibit PP2A should not reduce f-EPSP recovery after OGD. Consistent with this hypothesis, only high doses of OA had an effect on f-EPSP recovery. f-EPSP recovery was significantly reduced at $1 \mu \mathrm{M}$ OA but not at $1 \mathrm{nM}$, a concentration insufficient to inhibit PP1 (dose effect, $p<0.05$ ) (Fig. $1 C$; supplemental Fig. $1 A$, available at www.jneurosci.org as supplemental material). The $\mathrm{IC}_{50}$ for this effect was $53 \mathrm{~nm}$ (95\% confidence, 64.3 pM to $54.7 \mu \mathrm{M}$ ) (supplemental Fig. $1 B$, available at www.jneurosci.org as supplemental material), again suggesting a PP1-dependent f-EPSP recovery.

To confirm the PP1 dependence of the effect and delineate the cell type specificity of the functions of PP1, we used transgenic mice in which PP1 is selectively inhibited in forebrain neurons (to $67.7 \pm 12 \%$ ) by expression of a constitutively active form of the PP1 inhibitor, inhibitor-1 (I-1*) (Genoux et al., 2002; Michalon et al., 2005). Consistent with the reduced recovery observed with tautomycin and $\mathrm{OA}, \mathrm{I}-1^{*}$-mediated PP1 inhibition in the hippocampus significantly diminished f-EPSP recovery after OGD in transgenic slices (Fig. 1D). This effect was not attributable to a change in basal synaptic transmission, which was normal in transgenic slices (Jouvenceau et al., 2006). These results therefore confirm that suppression of recovery is attributable to PP1 inhibition, and indicate that the effect can be induced by selective inhibition of PP1 in neuronal cells.

\section{Induction of LTP before OGD mimics the effect of PP1 inhibition}

We next evaluated whether a change in PP1 activity induced by physiological stimulation can also modulate f-EPSP recovery after OGD. Previous studies have suggested that long-term changes in synaptic transmission are associated with alterations in protein phosphatase activity. Specifically, PP1 and PP2A activity is transiently inactivated shortly after induction of LTP but is activated shortly after induction of LTD (Winder and Sweatt, 2001). Therefore, we used LTP to attempt to decrease PP1 activity in area CA1 before OGD by stimulating Schaffer collaterals with a high-frequency tetanus (HFS; $100 \mathrm{~Hz}, 1 \mathrm{~s}$ ) in wild-type slices (LTP) (Fig. 1E). After LTP and OGD, f-EPSP slope decreased but recovered to a level that was significantly lower than in slices not subjected to LTP $(42.3 \pm 2.9 \%)$ (Fig. $1 E)$. This effect was specific to the potentiated synapses because simultaneous two-pathway recordings showed normal f-EPSP recovery in an adjacent nontetanized pathway (no LTP) (Fig. $1 E$, inset). We confirmed that the effect of LTP on recovery was associated with a change in PP1 activity by measuring PP1 and PP2A activity in slices subjected to LTP, both immediately ( $0 \mathrm{~min})$ and $1 \mathrm{~h}(1 \mathrm{~h})$ after OGD. OGD itself was found to rapidly diminish PP1 activity (no LTP after $1 \mathrm{~h}$, $56.4 \pm 12.4 \%$ inhibition from basal) (Fig. $1 F$ ). However, when LTP was induced before OGD, PP1 activity was decreased significantly more than without LTP $1 \mathrm{~h}$ after OGD. The decrease in 

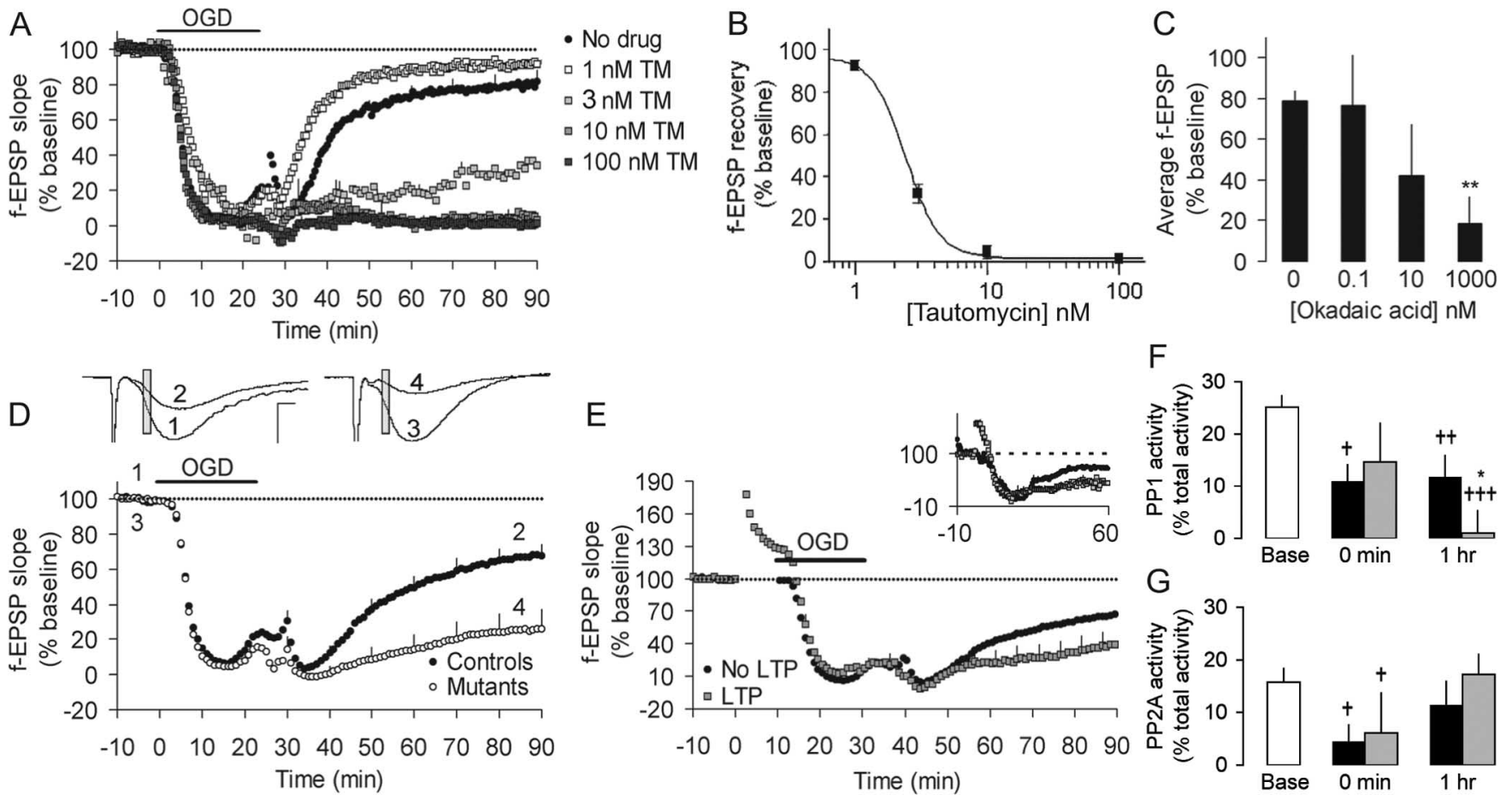

Figure 1. PP1 inhibition reducesf-EPSP recovery from OGD. $A$, Tautomycin (TM) diminishesf-EPSP recovery after OGD in a dose-dependent manner $\left(F_{(5,20)}=139.8 ; p<0.3\right)$. Mean $f$-EPSP slope 80 - 90 min after the onset of $0 \mathrm{GD}: 1 \mathrm{~nm}, 88.6 \pm 1.5 \%, \mathrm{NS}, n=5 ; 3 \mathrm{~nm}, 31.4 \pm 4.6 \%, p<0.001, n=5 ; 10 \mathrm{~nm}, 4.4 \pm 2.9 \%, p<0.001, n=3 ; 100 \mathrm{~nm}, 0.9 \pm 1.3 \%, p<0.001, n=5 ;$ versus control slices $(n=4) 79.3 \pm 6.2 \%, p=0.01 . B$, I $C_{50}$ for this effect is $2.4 \mathrm{~nm}(95 \%$ confidence, $2.2-2.7 \mathrm{~nm})$. C, Dose-dependent reduction of f-EPSP $\left(F_{(5,29)}=3.54 ; p<0.05\right)$ after okadaic acid treatment. After $80-90$ min, f-EPSP slope recovers up to $76.6 \pm 20.5 \%, \mathrm{NS}, n=4$ at $0.1 \mathrm{~nm}, 56.6 \pm 23.9 \%, \mathrm{NS}, n=4$ at $10 \mathrm{~nm}$, and $20.3 \pm 14.4 \%, p<0.001, n=8$ at $1 \mu \mathrm{m}$ versus $0 \mathrm{~nm}$ control slices, $79.2 \pm$ $4.9 \%, p<0.005, n=10,{ }^{* *} p<0.001$ versus $0 G D$. D , Genetic inhibition of PP1 in $1-1^{*}$ mutant mice reduces f-EPSP recovery after $0 G D$ down to $34.2 \pm 9.7 \%$ of baseline 90 min after the onset of OGD compared with $71.3 \pm 5.8 \%$ in control slices (mean effect of genotype, $F_{(1,28)}=11.81 ; p<0.005 ;$ controls, $n=13 ;$ mutants, $n=17$ ). The inset shows representative traces before (1,3) and after $(2,4)$ OGD in control $(1,2)$ and mutant $(3,4)$ slices (scale: $x, 2 \mathrm{~ms} ; y, 0.8 \mathrm{mV})$. $E$, LTP induced $10 \mathrm{~min}$ before 0 GD reduces $f$-EPSP slope recovery in control hippocampal slices. Mean $f$-EPSP slope (over the last 10 min of recording) in 0GD with no LTP $(69.3 \pm 3.5 \% ; n=33)$ is significantly different from LTP $(40.6 \pm 13.7 \% ; n=11)$ Tukey's post hoc, $p<0.005$. Inset shows a two-pathway experiment in which f-EPSP recovery is diminished only in potentiated synapses (no LTP pathway: $70.0 \pm 3.5 \%, n=4$, versus LTP pathway: $37.5 \pm 3.5 \%, n=4$, Tukey's post hoc, $p<0.001$ ). $\boldsymbol{F}$, PP1 activity (percent total protein phosphatase activity) before 0GD (white bar), and immediately ( $0 \mathrm{~min}$ ) or $1 \mathrm{~h}$ ( $1 \mathrm{~h}$ ) after 0 GD in slices not subjected to LTP (black bars) or preceded by LTP (gray bars). OGD, 0 min: $F_{(3,44)}=2.947, p<0.05 ;$ no LTP: $56.4 \pm 12.4 \%$ inhibition from baseline, $n=10$, LSD post hoc, $p<0.05 ; 0 G D 1$ h: $F_{(3,38)}=14.98, p<0.001$; No LTP: 52.9 $\pm 15.9 \%$ inhibition from baseline, $n=8$, Tukey's post hoc, $p<0.05$; and LTP/OGD 1 h: $99.5 \pm 17.0 \%$ inhibition from baseline, $n=9$, Tukey's post hoc, $p<0.001$ compared with baseline, LSD post hoc, $p<0.05$ compared with no LTP OGD $1 \mathrm{~h}$. Statistical significance compared with baseline: ${ }^{\dagger} p<0.05,{ }^{+\dagger} p<0.01,{ }^{\mathrm{t} t} p<0.001$; compared with no LTP: ${ }^{*} p<0.05$. G, PP2A activity is not altered by LTP induced before OGD (OGD 0 min: no LTP, $4.5 \pm 3.1, n=7 ;$ LTP, $6.1 \pm 7.6, n=6$, vs OGD 1 h: no LTP, $11.5 \pm 4.4 \%, n=7 ;$ LTP, $17.2 \pm 3.9 \%$ ). Black bars, No LTP; gray bars, subjected to LTP.

f-EPSP recovery was again specific to PP1 because PP2A, also inhibited by OGD alone, was not further inhibited by LTP induction (Fig. 1G).

\section{Induction of LTD before OGD promotes recovery}

We then tested the effect of LTD induction on f-EPSP recovery. Remarkably, in contrast to LTP, LTD induction led to a full recovery of f-EPSP, which, after OGD, reached a level comparable with that in slices subjected to LTD without OGD (Fig. 2A). Two-pathway recordings confirmed that the beneficial effect of LTD was specific to depressed synapses (Fig. 2A, inset). Furthermore, it was accompanied by full restoration of PP 1 activity $1 \mathrm{~h}$ after OGD, an effect that was not observed in the absence of LTD (Fig. 2 B). It was specific to PP1, because PP2A activity was not significantly changed by LTD induction (Fig. $2 C$ ). To confirm that the full recovery of f-EPSP induced by LTD is mediated by PP1, we repeated the experiment in hippocampal slices from $\mathrm{I}-1^{\star}$ transgenic mice. In these slices, partial inhibition of PP1 [67.7 $\pm 12 \%$ (Genoux et al., 2002)] did not abolish the beneficial effect of LTD on f-EPSP recovery (Fig. 2D, LTD mutants), most likely because of the residual PP1 activity not fully inhibited by $\mathrm{I}-1^{\star}$ expression. Consistent with this hypothesis, blockade of this residual activity by a suboptimal dose of tautomycin (1 nM) fully abolished the beneficial effect of LTD on f-EPSP recovery (Fig. $2 \mathrm{D}$, LTD mutant/tautomycin). This dose of tautomycin had no effect on LTD in control slices (Fig. 2 E, inset) but abolished LTD in I- $1^{*}$ slices (Fig. 2E). These results highlight a neuroprotective role for LTD through PP1-dependent mechanisms.

\section{Genetic inhibition of PP1 aggravates injury after focal cerebral ischemia in vivo and involves ERK-dependent pathways}

To validate our findings in vivo, we next examined whether the genetic inhibition of PP1 in adult mice affects the extent of damage after ischemia. I- $1^{\star}$ transgenic mice and control littermates were subjected to intraluminal MCAO, and the effect on brain tissue was evaluated $24 \mathrm{~h}$ later. Consistent with our findings in vitro, PP1 inhibition significantly enlarged infarct volume in I-1* mutant mice (Fig. $3 A, B$ ) and increased TUNEL staining, indicating a larger number of cells undergoing cell death (Fig. $3 D, E$ ). This effect was not attributable to impaired blood circulation because Doppler flowmetry showed similar hemodynamic changes in control and transgenic mice during MCAO (Fig. $3 C, F)$. Because the degree of cell survival after ischemia depends on the activation or suppression of apoptotic pathways, we next 
examined whether major apoptotic processes are associated with the detrimental effect of PP1 inhibition. Western blot analyses revealed that the phosphorylation level of ERK1, ERK2, and JNK2, cytoplasmic signaling molecules involved in ischemia-induced cell death (Bogoyevitch et al., 2004; Chu et al., 2004) (but see Hetman and Gozdz, 2004), were significantly increased by PP1 inhibition in vivo (Fig. $4 A, C)$. Remarkably, after MCAO, phosphorylated ERK1 and ERK2 were further increased in $\mathrm{I}-1^{*}$ mutant mice (Fig. $4 A, C$ ). Importantly, this effect was confirmed in vitro after OGD and PP1 inhibition (Fig. $4 B$ ), indicating that similar mechanisms are recruited in vivo and in vitro. The consistency of these results strongly suggests that ERK1/2 activation is regulated by PP1 during an ischemic insult and that PP1 converges onto similar cell survival/death pathway in both models. Finally, decreased expression of the antiapoptotic protein $\mathrm{Bcl}-\mathrm{X}_{\mathrm{L}}$ and activation of the apoptotic protease caspase- 3 induced by MCAO were accentuated by genetic inhibition of PP1 in vivo (Fig. $4 E, F$ ), pointing to the potential involvement of these opposing pathways in PP1-dependent mechanisms.

\section{Discussion}

The present results provide evidence that the serine/threonine protein phosphatase PP1 and PP1-dependent bidirectional plasticity are critical for the mechanisms of recovery from excitotoxicity in the adult mouse brain. These results demonstrate that a decrease in PP1 activity induced by pharmacological or genetic inhibition before OGD in hippocampal slices or before MCAO in vivo, alters the mechanisms of recovery. Furthermore, in vitro f-EPSP recovery, a parameter affected by cellular damage, is impaired when LTP is induced before OGD, an effect that is associated with selective inhibition of PP1. In contrast, the induction of LTD before OGD promotes full f-EPSP recovery and is associated with restoration of PP1 activity after OGD. Consistently, the beneficial effect of LTD is abolished by blockade of PP1 activity. These results confirm that recovery from OGD involves PP1-dependent pathways and indicate that modulation of recovery by synaptic plasticity is associated with direct changes in PP1 activity. Several targets of apoptotic and cell survival pathways are involved in the detrimental effect of PP1 inhibition on ischemic injury. PP1 inhibition is accompanied by increased phosphorylation of ERK1/2 both in vitro and in vivo, and increased JNK2 phosphorylation, caspase-3 activation, and decreased $\mathrm{Bcl}-\mathrm{X}_{\mathrm{L}}$ expression in vivo. Together, these findings reveal a novel molecular mechanism for brain recovery that depends on PP1 and highlight a yet-unknown function for LTP and LTD in regulating the brain response to injury.

Protein serine/threonine phosphatases have been suggested to promote neuroprotection (Fernandez et al., 1993; Nuydens et al., 1998; Runden et al., 1998; Klumpp and Kriegl- stein, 2002; Stevens et al., 2003; Yi et al., 2005), but the specific involvement of PP1 could not be examined until now because of the limited specificity of pharmacological inhibitors and the lack of selective activators of PP1. The only finding specific for PP1 after ischemia showed rather contradictory results revealing increased PP $1 \alpha$ and PP1 $\gamma$ mRNA in the hippocampal CA1 region and the striatum, but decreased $\mathrm{PP} 1 \gamma$ protein and no change in PP1 $\alpha$ (Horiguchi et al., 2002). In the present study, the combination of pharmacological, genetic, and physiological methods allowed us to specifically manipulate PP1 activity before experimental ischemia both in vitro and in vivo, and demonstrate that PP1 is involved in the mechanisms of recovery from excitotoxicity. Our manipulation further provided new evidence that ERK1/2 expression and phosphorylation are regulated by $\mathrm{PP} 1$ during an ischemic insult, both in vitro and in vivo. Many reports using models of cerebral ischemia, brain trauma, or neurodegenerative diseases have demonstrated a detrimental role for ERK1/2 and JNK1/2 signaling (Irving and Bamford, 2002; Bogoyevitch et al., 2004; Chu et al., 2004). However, ERK1/2 has also been shown to be neuroprotective, suggesting that the function of ERK1/2 may depend on the nature, duration, and severity of injury, cell type, and brain area, or timing of analysis (Hetman and Gozdz, 2004). In the 
A

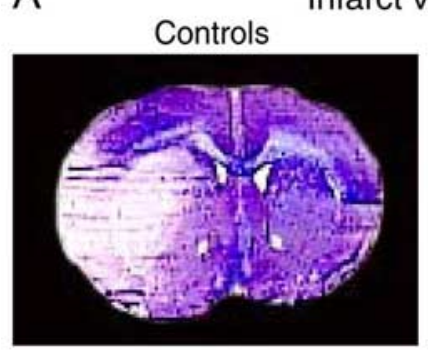

Infarct volume

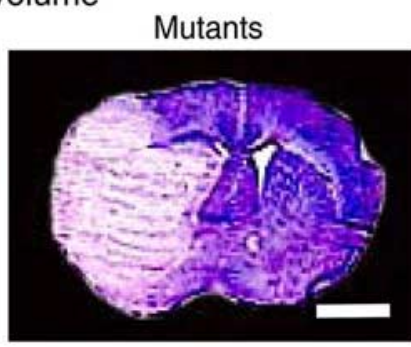

B
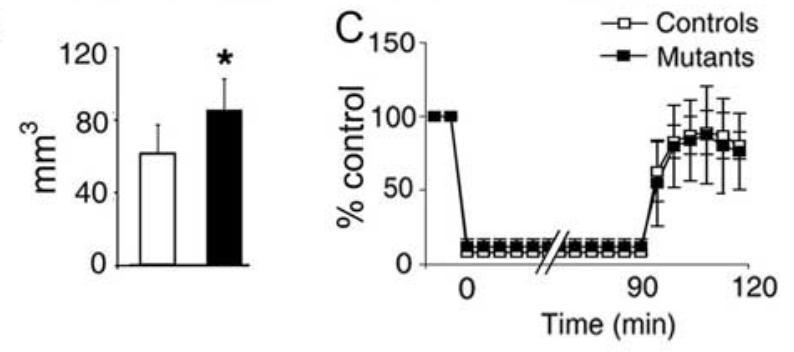

D

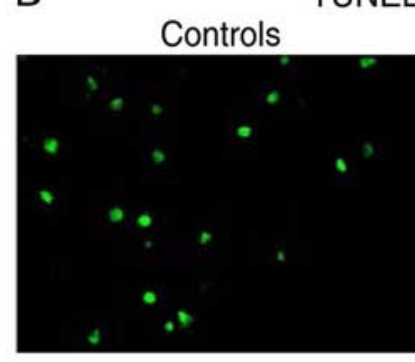

TUNEL staining

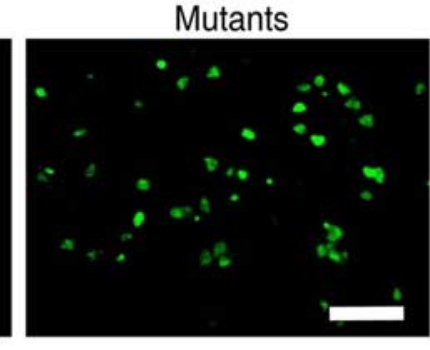

$\mathrm{E}$

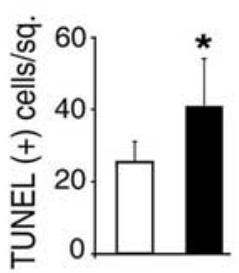

$\mathrm{F}$

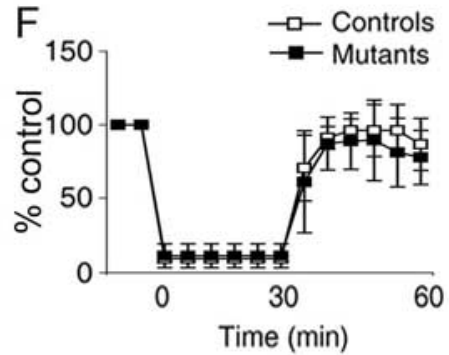

Figure 3. Genetic inhibition of PP1 aggravates brain infarct and disseminates neural injury after MCAO. $A, B$, Representative cresyl violet staining of coronal brain sections $(A)$ and bar graph indicating mean infarct volume $(\boldsymbol{B}) 24 \mathrm{~h}$ after $90 \mathrm{~min} \mathrm{MCAO}$ and reperfusion in $1-1^{*}$ mutant mice and control littermates (two-tailed $t$ test, $p<0.05 ; n=8 /$ group). Scale bar, $2 \mathrm{~mm}$. $\boldsymbol{D}, \boldsymbol{E}$, Representative TUNEL staining $(\boldsymbol{D})$ and bar graph indicating mean density of DNA-fragmented cells $(\boldsymbol{E})$ in striatum $72 \mathrm{~h}$ after $30 \mathrm{~min}$ MCA 0 and reperfusion in I-1* mutant mice and control littermates (two-tailed $t$ test, $p<0.05 ; n=6 /$ group). Scale bar, $100 \mu \mathrm{m}$. $\boldsymbol{C}, \boldsymbol{F}$, Laser Doppler flowmetry during $90 \mathrm{~min}(\boldsymbol{C})$ or $30 \mathrm{~min}(\boldsymbol{F})$ MCA0 followed by 30 min reperfusion indicating similar level of perfusion in $\mathrm{I}-1^{*}$ mutant mice and control littermates. ${ }^{*} p<0.05$.

postischemic brain, the level of phosphorylated, activated ERK1/2 and JNKs was reported to rapidly increase (Farrokhnia et al., 2005; Shackelford and Yeh, 2006), and inhibition of ERK1/2 or JNKs suppresses neuronal injury after transient or permanent MCAO in the rat (Alessandrini et al., 1999; Borsello et al., 2003). However, the actual pathways linking the initial steps of excitotoxicity to ERK/JNK activation and apoptotic processes remain poorly understood.

Our results now provide evidence that PP1 is involved in the regulation of ERK1/2 and JNK2 pathways and critically determines the response of neurons to excitotoxicity that engage these pathways both in vitro and in vivo. This central function of PP1 suggests that it is strategically positioned upstream of major apoptotic/survival pathways, consistent with its ability to be retained at the membrane near glutamate receptors through scaffolding proteins (Westphal et al., 1999). There, PP1 may couple the NMDA receptor to downstream ERKs, JNKs, and apoptotic pathways as suggested by our correlated data between PP1 activity, ERK1/2 phosphorylation and recovery. It may dephosphorylate ERK1/2 directly (Quevedo et al., 2003) or indirectly possibly by downregulating upstream MEKs such as reported in rat pituitary cells (Manfroid et al., 2001). Furthermore, the simultaneous increase in ERK1/2 and JNK2 phosphorylation induced by PP1 inhibition in vivo suggests that PP1 may, in addition, act to coordinate ERK1/2 and JNK2 pathways. This highlights a yetunknown PP1-dependent cross talk between MAPK and JNK pathways that may operate a few hours after injury (not observed $1 \mathrm{~h}$ after OGD in vitro). The ability of PP1 to associate with JNK2 in a tripartite complex in mitochondria may underlie this feature, and provide a means to tightly control this interaction (Brichese et al., 2004). Moreover, the concomitant activation of ERK1/2 and JNK2 may exacerbate the impact of excitotoxicity by activating the protease caspase-3. JNK1/2 activation is known to induce the translocation of the proapoptotic protein Bax to mitochondria. This operates by release of Bax from cytoplasmic sequestration and cleavage of procaspase- 3 into activated caspase- 3 (Guan et al., 2006). Caspase-3-mediated apoptosis was indeed reported to be induced by PP1/PP2A inhibitors in several cell types in culture (Fladmark et al., 1999). In turn, activated caspase-3 may cleave antiapoptotic proteins such as $\mathrm{Bcl}-\mathrm{X}_{\mathrm{L}}$ and Bcl-2, both of which are known to associate with PP1 through the RVXF PP1-binding motif (Ayllon et al., 2000). Thus, overall, the simultaneous upregulation of apoptotic proteins and downregulation of antiapoptotic molecules induced by PP1 inhibition in vivo indicate that PP1 has an impact on multiple targets and pathways to limit excitotoxicity. The consistent link between PP1 and ERK1/2 that we showed both in vitro and in vivo also strongly suggests that the mechanisms of recovery in these two systems share dependence on these two targets; however, it does not rule out the possibility that additional mechanisms are also recruited.

Our findings that two major forms of synaptic plasticity in vitro, LTP and LTD, modulate recovery from an OGD insult reveal a novel physiological function for bidirectional hippocampal plasticity in brain repair and damage. The correlation between their opposite effect on recovery and on PP1 activity strongly indicates that both LTP and LTD operate through PP1dependent pathways to modulate recovery. These results therefore point to a pivotal role for PP1 not only in the control of bidirectional plasticity such as previously demonstrated (Blitzer et al., 1998; Morishita et al., 2001; Jouvenceau et al., 2006) but also in the control of plasticity-dependent modulation of brain recovery. This pivotal role may again derive from the strategic positioning of PP1 in neuronal cells, in the vicinity of major neurotransmitter receptor complexes such as the NMDA receptor complex known to be critical for excitotoxicity (Lisman and Zhabotinsky, 2001; Colbran, 2004; Mansuy and Shenolikar, 2006). Furthermore, the demonstration that LTP or LTD before OGD is detrimental or beneficial to recovery suggests that previous activity at synapses has a strong influence on the response to injury. This finding underscores the critical importance of the state of plasticity of synaptic circuits at the time of insult, a notion reminiscent of metaplasticity. Notably, a form of synaptic depression was previously suggested to enhance survival after transient OGD in a population of hippocampal CA1 neurons in vivo 
A
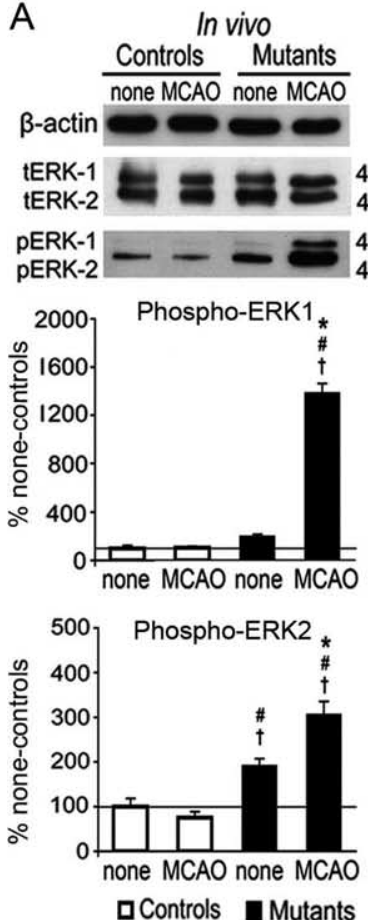

B
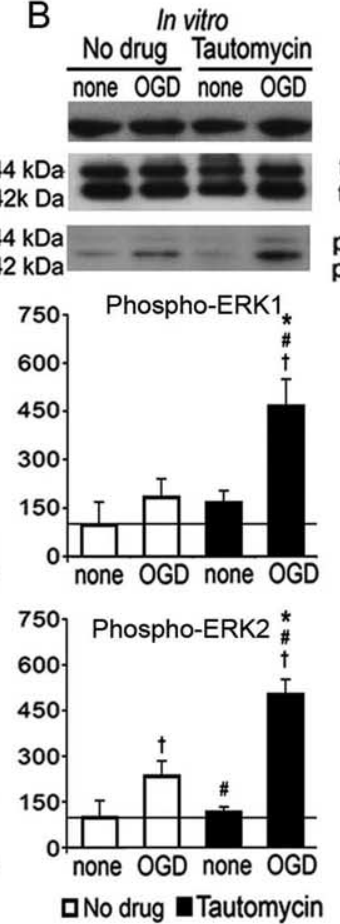
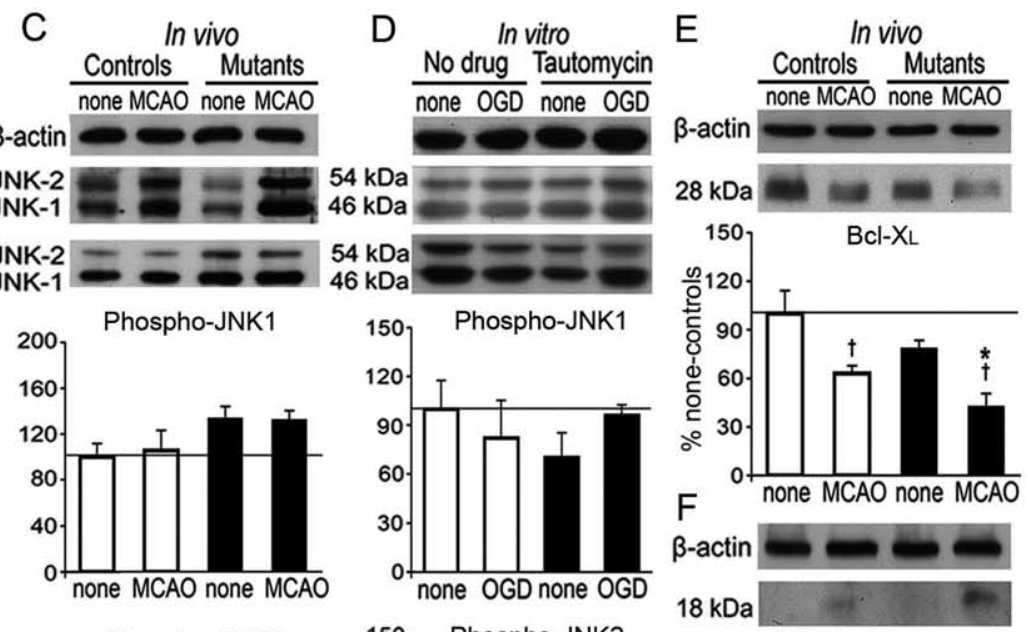

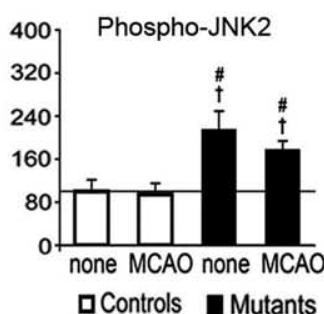

$54 \mathrm{kDa}$
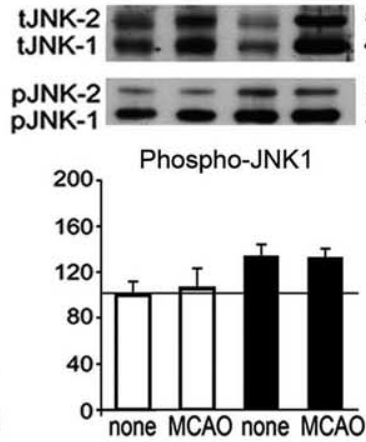

controls Mutants
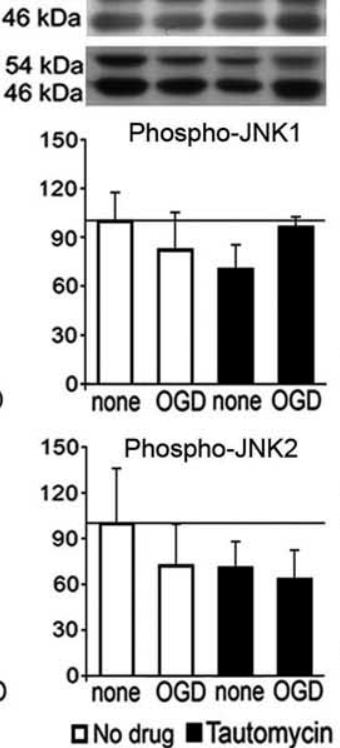

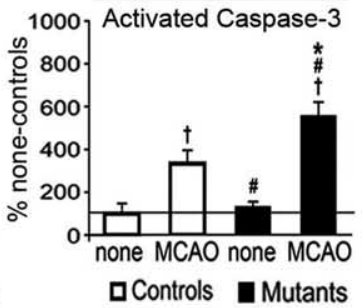

Figure 4. PP1 inhibition increases ERK1/2 phosphorylation after MCAO in vivo and OGD in vitro. Western blots (top panels) and quantified bar graphs (middle and bottom panels) for contralateral (none) and ipsilateral (MCAO) homogenates from striatum in I-1* mutant mice (black bars) and control littermates (white bars) after 30 min MCA0 and $72 \mathrm{~h}$ reperfusion ( $n=6 /$ group) showing ( $A$ ) total and phosphorylated ERK1 ( $+98.1 \pm 22.3 \%$; ANOVA/LSD post hoc, $p<0.05 ; n=3 /$ group) and ERK2 ( $+91.0 \pm 17.8 \%$; ANOVA/LSD post hoc, $p<0.05 ; n=3 /$ group) and $(C)$ total and phosphorylated JNK1 ( $+33.4 \pm 10.8 \%$; ANOVA/LSD posthoc, NS; $n=3$ /group) and JNK2 $\left(+114.1 \pm 35.3 \%\right.$; ANOVA/LSD posthoc, $p<0.05 ; n=3 /$ group). MCA0 in I- ${ }^{*}$ mutant micedramatically increases the level of phosphorylated ERK1 ( $+599.0 \pm 42.2 \%$; ANOVA/LSD post hoc, $p<0.05 ; n=3 /$ group) and ERK2 ( $+60.1 \pm 16.0 \%$; ANOVA/LSD post hoc, $p<0.05 ; n=3 /$ group) (but not JNK1 or -2). $\boldsymbol{B}, \boldsymbol{D}$, Slices from control mice subjected to OGD (OGD) or no OGD (none) and treated with $3 \mathrm{~nm}$ tautomycin (black bars) or vehicle (white bars). PP1 inhibition results in higher level of phosphorylated ERK1/2 after OGD than in control slices (ERK1: $+366.8 \pm 48.6 \%$; ANOVA/LSD test, $p<0.05 ; n=3$; ERK2: $+404.1 \pm 28.5 \%$; ANOVA/LSD post hoc, $p<0.05 ; n=3$ ). In vitro, PP1 inhibition alone does not increase phosphorylated JNK2 (D), perhaps because of shorter PP1 inhibition compared with in vivo (a few hours for in vitro tautomycin treatment vs several days of I- F $^{*}$ expression in the mutant mice). $\boldsymbol{E}, \boldsymbol{F}$, Western blots (top panels) and quantified bar graphs (middle and bottom panels) showing decreased $B C-X_{L}$ expression and increased caspase-3 activation after MCA0 in I- * $^{*}$ mutant mice. BCI-X, is decreased by $-49.0 \pm 13.5 \%$ (ANOVA/LSD post hoc, $p<0.05 ; n=3 /$ group); activated caspase-3 is increased by $+60.2 \pm 19.7 \%$ (ANOVA/LSD post hoc, $p<$ $0.05 ; n=3 /$ group).

(Papas et al., 1993; Gao et al., 1998). In a similar preparation, neurons more likely to die have been found to exhibit a form of potentiation that may be similar to anoxic LTP reported in vitro after short periods of OGD that may act as a prelude to injury (Hori and Carpenter, 1994; Calabresi et al., 2003). These findings corroborate our demonstration that LTP is neurotoxic and LTD neuroprotective. However, it should also be noted that LTP has been suggested to confer protection during preconditioning when induced in between short episodes of hypoxia, through mechanisms involving $A_{1}$ adenosine receptors (Youssef et al., 2001, 2003). However, in these studies, brief hypoxia was also found to decrease synaptic responses (Youssef et al., 2001) and downregulate $\mathrm{Ca}^{2+}$ signaling, CaMKII, and PKC (Katsura et al., 2001), which interfered with LTP induction, suggesting that neuroprotection may not directly derive from LTP itself (Youssef et al., 2006). Finally, attenuation of kainic acid-induced neuronal loss by LTP was also reported, suggesting that LTP may have neuroprotective properties in certain conditions (Evans and Wang, 2005).

Last, this study advances the understanding of the mechanisms of damage and protection in the context of ischemia and may provide new perspectives for the potential development of treatment against brain pathologies such as ischemic stroke, traumatic brain injury, or some neurodegenerative disorders.

\section{References}

Aarts MM, Tymianski M (2003) Novel treatment of excitotoxicity: targeted disruption of intracellular signalling from glutamate receptors. Biochem Pharmacol 66:877-886.

Alessandrini A, Namura S, Moskowitz MA, Bonventre JV (1999) MEK1 protein kinase inhibition protects against damage resulting from focal cerebral ischemia. Proc Natl Acad Sci USA 96:12866-12869.

Aronowski J, Grotta JC, Strong R, Waxham MN (2000) Interplay between the gamma isoform of PKC and calcineurin in regulation of vulnerability to focal cerebral ischemia. J Cereb Blood Flow Metab 20:343-349.

Arundine M, Tymianski M (2003) Molecular mechanisms of calciumdependent neurodegeneration in excitotoxicity. Cell Calcium 34:325-337.

Arundine M, Tymianski M (2004) Molecular mechanisms of glutamatedependent neurodegeneration in ischemia and traumatic brain injury. Cell Mol Life Sci 61:657-668.

Asai A, Qiu J, Narita Y, Chi S, Saito N, Shinoura N, Hamada H, Kuchino Y, Kirino T (1999) High level calcineurin activity predisposes neuronal cells to apoptosis. J Biol Chem 274:34450-34458.

Ayllon V, Martinez AC, Garcia A, Cayla X, Rebollo A (2000) Protein phosphatase lalpha is a Ras-activated Bad phosphatase that regulates interleukin-2 deprivation-induced apoptosis. EMBO J 19:2237-2246.

Blitzer RD, Connor JH, Brown GP, Wong T, Shenolikar S, Iyengar R, Landau EM (1998) Gating of CaMKII by cAMP-regulated protein phosphatase activity during LTP. Science 280:1940-1942.

Bogoyevitch MA, Boehm I, Oakley A, Ketterman AJ, Barr RK (2004) Tar- 
geting the JNK MAPK cascade for inhibition: basic science and therapeutic potential. Biochim Biophys Acta 1697:89-101.

Borsello T, Clarke PG, Hirt L, Vercelli A, Repici M, Schorderet DF, Bogousslavsky J, Bonny C (2003) A peptide inhibitor of c-Jun N-terminal kinase protects against excitotoxicity and cerebral ischemia. Nat Med 9:1180-1186.

Brichese L, Cazettes G, Valette A (2004) JNK is associated with Bcl-2 and PP1 in mitochondria: paclitaxel induces its activation and its association with the phosphorylated form of Bcl-2. Cell Cycle 3:1312-1319.

Calabresi P, Centonze D, Pisani A, Cupini L, Bernardi G (2003) Synaptic plasticity in the ischaemic brain. Lancet Neurol 2:622-629.

Chu CT, Levinthal DJ, Kulich SM, Chalovich EM, DeFranco DB (2004) Oxidative neuronal injury. The dark side of ERK1/2. Eur J Biochem 271:2060-2066

Colbran RJ (2004) Protein phosphatases and calcium/calmodulindependent protein kinase II-dependent synaptic plasticity. J Neurosci 24:8404-8409.

Evans GL, Wang Y (2005) LTP induction prevents neurodegeneration in the CA1 region of the hippocampus in rats exposed to kainic acid in vivo. Soc Neurosci Abstr 31:967.6.

Farrokhnia N, Roos MW, Terent A, Lennmyr F (2005) Differential early mitogen-activated protein kinase activation in hyperglycemic ischemic brain injury in the rat. Eur J Clin Invest 35:457-463.

Fernandez MT, Zitko V, Gascon S, Torreblanca A, Novelli A (1993) Neurotoxic effect of okadaic acid, a seafood-related toxin, on cultured cerebellar neurons. Ann NY Acad Sci 679:260-269.

Fladmark KE, Brustugun OT, Hovland R, Boe R, Gjertsen BT, Zhivotovsky B, Doskeland SO (1999) Ultrarapid caspase-3 dependent apoptosis induction by serine/threonine phosphatase inhibitors. Cell Death Differ 6:1099-1108.

Fowler JC (1989) Adenosine antagonists delay hypoxia-induced depression of neuronal activity in hippocampal brain slice. Brain Res 490:378-384

Gao TM, Pulsinelli WA, Xu ZC (1998) Prolonged enhancement and depression of synaptic transmission in CA1 pyramidal neurons induced by transient forebrain ischemia in vivo. Neuroscience 87:371-383.

Genoux D, Haditsch U, Knobloch M, Michalon A, Storm D, Mansuy IM (2002) Protein phosphatase 1 is a molecular constraint on learning and memory. Nature 418:970-975.

Guan QH, Pei DS, Xu TL, Zhang GY (2006) Brain ischemia/reperfusioninduced expression of DP5 and its interaction with Bcl-2, thus freeing Bax from Bcl-2/Bax dimmers are mediated by c-Jun $\mathrm{N}$-terminal kinase (JNK) pathway. Neurosci Lett 393:226-230.

Gupta V, Ogawa AK, Du X, Houk KN, Armstrong RW (1997) A model for binding of structurally diverse natural product inhibitors of protein phosphatases PP1 and PP2A. J Med Chem 40:3199-3206.

Hermann DM, Kilic E, Kugler S, Isenmann S, Bahr M (2001) Adenovirusmediated GDNF and CNTF pretreatment protects against striatal injury following transient middle cerebral artery occlusion in mice. Neurobiol Dis 8:655-666.

Hetman M, Gozdz A (2004) Role of extracellular signal regulated kinases 1 and 2 in neuronal survival. Eur J Biochem 271:2050-2055.

Hori N, Carpenter DO (1994) Functional and morphological changes induced by transient in vivo ischemia. Exp Neurol 129:279-289.

Horiguchi T, Shima H, Suga S, Ogino M, Shimizu K, Toya S, Nagao M, Kawase $\mathrm{T}$ (2002) Transient forebrain ischemia induces expression of serine/threonine protein phosphatase $1 \mathrm{mRNA}$ in the vulnerable regions of gerbil brain. Neurosci Lett 325:115-118.

Irving EA, Bamford M (2002) Role of mitogen- and stress-activated kinases in ischemic injury. J Cereb Blood Flow Metab 22:631-647.

Jouvenceau A, Hedou G, Potier B, Kollen M, Dutar P, Mansuy IM (2006) Partial inhibition of PP1 alters bidirectional synaptic plasticity in the hippocampus. Eur J Neurosci 24:564-572.

Katsura KI, Kurihara J, Kato H, Katayama Y (2001) Ischemic preconditioning affects the subcellular distribution of protein kinase $\mathrm{C}$ and calcium/calmodulin-dependent protein kinase II in the gerbil hippocampal CA1 neurons. Neurol Res 23:751-754.

Kilic E, Kilic U, Soliz J, Bassetti CL, Gassmann M, Hermann DM (2005) Brain-derived erythropoietin protects from focal cerebral ischemia by dual activation of ERK-1/-2 and Akt pathways. FASEB J 19:2026-2028.
Klumpp S, Krieglstein J (2002) Serine/threonine protein phosphatases in apoptosis. Curr Opin Pharmacol 2:458-462.

Legos JJ, Tuma RF, Barone FC (2002) Pharmacological interventions for stroke: failures and future. Expert Opin Investig Drugs 11:603-614.

Lipton P (1999) Ischemic cell death in brain neurons. Physiol Rev 79:1431-1568.

Lisman JE, Zhabotinsky AM (2001) A model of synaptic memory: a CaMKII/PP1 switch that potentiates transmission by organizing an AMPA receptor anchoring assembly. Neuron 31:191-201.

Lobner D, Lipton P (1993) Intracellular calcium levels and calcium fluxes in the CA1 region of the rat hippocampal slice during in vitro ischemia: relationship to electrophysiological cell damage. J Neurosci 13:4861-4871.

Lushnikova IV, Voronin KY, Malyarevskyy PY, Skibo GG (2004) Morphological and functional changes in rat hippocampal slice cultures after short-term oxygen-glucose deprivation. J Cell Mol Med 8:241-248.

Manfroid I, Martial JA, Muller M (2001) Inhibition of protein phosphatase $\mathrm{PP} 1$ in GH3B6, but not in GH3 cells, activates the MEK/ERK/c-fos pathway and the human prolactin promoter, involving the coactivator $\mathrm{CPB} /$ p300. Mol Endocrinol 15:625-637.

Mansuy IM, Shenolikar S (2006) Protein serine/threonine phosphatases in neuronal plasticity and disorders of learning and memory. Trends Neurosci 29:679-686.

Mansuy IM, Mayford M, Jacob B, Kandel ER, Bach ME (1998) Restricted and regulated overexpression reveals calcineurin as a key component in the transition from short-term to long-term memory. Cell 92:39-49.

Michalon A, Koshibu K, Baumgartel K, Spirig DH, Mansuy IM (2005) Inducible and neuron-specific gene expression in the adult mouse brain with the rtTA2S-M2 system. Genesis 43:205-212.

Morioka M, Hamada J, Ushio Y, Miyamoto E (1999) Potential role of calcineurin for brain ischemia and traumatic injury. Prog Neurobiol $58: 1-30$.

Morishita W, Connor JH, Xia H, Quinlan EM, Shenolikar S, Malenka RC (2001) Regulation of synaptic strength by protein phosphatase 1. Neuron 32:1133-1148.

Nuydens R, de Jong M, Van Den Kieboom G, Heers C, Dispersyn G, Cornelissen F, Nuyens R, Borgers M, Geerts H (1998) Okadaic acid-induced apoptosis in neuronal cells: evidence for an abortive mitotic attempt. J Neurochem 70:1124-1133.

Olney JW, Sharpe LG (1969) Brain lesions in an infant rhesus monkey treated with monosodium glutamate. Science 166:386-388.

Onodera H, Yamasaki Y, Kogure K, Miyamoto E (1995) Calcium/ calmodulin-dependent protein kinase II and protein phosphatase 2B (calcineurin) immunoreactivity in the rat hippocampus long after ischemia. Brain Res 684:95-98.

Papas S, Crepel V, Ben-Ari Y (1993) The NMDA receptor contributes to anoxic aglycemic induced irreversible inhibition of synaptic transmission. Brain Res 607:54-60.

Quevedo C, Salinas M, Alcazar A (2003) Initiation factor 2B activity is regulated by protein phosphatase 1 , which is activated by the mitogenactivated protein kinase-dependent pathway in insulin-like growth factor 1-stimulated neuronal cells. J Biol Chem 278:16579-16586.

Quintana P, Alberi S, Hakkoum D, Muller D (2006) Glutamate receptor changes associated with transient anoxia/hypoglycaemia in hippocampal slice cultures. Eur J Neurosci 23:975-983.

Raley-Susman KM, Lipton P (1990) In vitro ischemia and protein synthesis in the rat hippocampal slice: the role of calcium and NMDA receptor activation. Brain Res 515:27-38.

Runden E, Seglen PO, Haug FM, Ottersen OP, Wieloch T, Shamloo M, Laake JH (1998) Regional selective neuronal degeneration after protein phosphatase inhibition in hippocampal slice cultures: evidence for a MAP kinase-dependent mechanism. J Neurosci 18:7296-7305

Sattler R, Tymianski M (2000) Molecular mechanisms of calciumdependent excitotoxicity. J Mol Med 78:3-13.

Shackelford DA, Yeh RY (2006) Modulation of ERK and JNK activity by transient forebrain ischemia in rats. J Neurosci Res 83:476-488.

Sharkey J, Butcher SP (1994) Immunophilins mediate the neuroprotective effects of FK506 in focal cerebral ischaemia. Nature 371:336-339. 
Sharkey J, Crawford JH, Butcher SP, Marston HM (1996) Tacrolimus (FK506) ameliorates skilled motor deficits produced by middle cerebral artery occlusion in rats. Stroke 27:2282-2286.

Spudich A, Kilic E, Xing H, Kilic U, Rentsch KM, Wunderli-Allenspach H, Bassetti CL, Hermann DM (2006) Inhibition of multidrug resistance transporter-1 facilitates neuroprotective therapies after focal cerebral ischemia. Nat Neurosci 9:487-488.

Stevens TR, Krueger SR, Fitzsimonds RM, Picciotto MR (2003) Neuroprotection by nicotine in mouse primary cortical cultures involves activation of calcineurin and L-type calcium channel inactivation. J Neurosci 23:10093-10099.

Sugawara T, Fujimura M, Noshita N, Kim GW, Saito A, Hayashi T, Narasimhan P, Maier CM, Chan PH (2004) Neuronal death/survival signaling pathways in cerebral ischemia. NeuroRx 1:17-25.

Wang HG, Pathan N, Ethell IM, Krajewski S, Yamaguchi Y, Shibasaki F, McKeon F, Bobo T, Franke TF, Reed JC (1999) $\mathrm{Ca}^{2+}$-induced apoptosis through calcineurin dephosphorylation of BAD. Science 284:339-343.
Westphal RS, Tavalin SJ, Lin JW, Alto NM, Fraser ID, Langeberg LK, Sheng M, Scott JD (1999) Regulation of NMDA receptors by an associated phosphatase-kinase signaling complex. Science 285:93-96.

Winder DG, Sweatt JD (2001) Roles of serine/threonine phosphatases in hippocampal synaptic plasticity. Nat Rev Neurosci 2:461-474.

Yi KD, Chung J, Pang P, Simpkins JW (2005) Role of protein phosphatases in estrogen-mediated neuroprotection. J Neurosci 25:7191-7198.

Youssef FF, Addae JI, McRae A, Stone TW (2001) Long-term potentiation protects rat hippocampal slices from the effects of acute hypoxia. Brain Res 907:144-150.

Youssef FF, Addae JI, Stone TW (2003) LTP-induced depression of response to hypoxia in hippocampus: effects of adenosine receptor activation. NeuroReport 14:1809-1814.

Youssef FF, Addae JI, Stone TW (2006) NMDA-induced preconditioning attenuates synaptic plasticity in the rat hippocampus. Brain Res 10731074:183-189. 